\title{
Necessary and Sufficient Conditions for the Existence of a Hermitian Positive Definite Solution of a Type of Nonlinear Matrix Equations
}

\author{
Wenling Zhao, ${ }^{1}$ Hongkui Li, ${ }^{1}$ Xueting Liu, ${ }^{2}$ and Fuyi $\mathrm{Xu}^{1}$ \\ ${ }^{1}$ College of Science, Shandong University of Technology, Zibo 255049, Shandong, China \\ 2 School of Electrical and Electronic Engineering, Shandong University of Technology, \\ Zibo 255049, Shandong, China \\ Correspondence should be addressed to Wenling Zhao, zwlsdj@163.com
}

Received 13 February 2009; Revised 5 June 2009; Accepted 31 July 2009

Recommended by Victoria Vampa

We study the Hermitian positive definite solutions of the nonlinear matrix equation $X+A^{*} X^{-2} A=$ $I$, where $A$ is an $n \times n$ nonsingular matrix. Some necessary and sufficient conditions for the existence of a Hermitian positive definite solution of this equation are given. However, based on the necessary and sufficient conditions, some properties and the equivalent equations of $X+A^{*} X^{-2} A=I$ are presented while the matrix equation has a Hermitian positive definite solution.

Copyright (c) 2009 Wenling Zhao et al. This is an open access article distributed under the Creative Commons Attribution License, which permits unrestricted use, distribution, and reproduction in any medium, provided the original work is properly cited.

\section{Introduction}

In this paper, we conside the Hermitian positive definite solutions of the matrix equation:

$$
X+A^{*} X^{-2} A=I
$$

where $A$ is an $n \times n$ nonsingular matrix, $I$ denotes the identity matrix, and $A^{*}$ the conjugate transpose of $A$.

In many physical applications [1] (such as super-resolution image restoration, a new image processing branch, which restores one better image of higher resolution from a degraded image sequence of lower resolution, algorithms of super-resolution image restoration can be used wherever image resolution is not contented, and image sequence can be obtained), we must solve a system of linear equations [2]:

$$
M x=f,
$$


where the positive definite matrix $M$ arises from a finite different approximation to an elliptic partial differential equation. The solving of the System $M x=f$ can be transformed to the solving of the equations $X \pm A^{*} X^{-2} A=I$, and the nonlinear matrix equation (1.1), appears in such many applications including control theory, ladder networks, dynamic programming, stochastic filtering, statistic, and super-resolution image restoration. For such decomposition to exist the matrix $X$ must be a positive definite solution to the matrix equations $X+A^{*} X^{-2} A=$ $I$.

We know that the study of the Hermitian positive definite solutions of the matrix equation has three basic problems, the theoretic issue on solvability; the numerical solution; the analysis of the perturbation.

Because of the wide mathematical and physical background, in recent years, the existence of Hermitian positive solutions for nonlinear matrix equation received wide attention (1.1) [3], for various values of $q$. In [3], Ivanov et al. construct iterative methods for obtaining positive definite solutions of the matrix equation (1.1) and give some sufficient conditions for the existence of a positive definite solution. Moreover, Ferrante and Levy [4] studied the equation $X=Q+N X^{-1} N^{*}$, and presented an algorithm which converges to the positive definite solution for a wide range of conditions. Cheng [5] presented some sufficient conditions and new necessary conditions for the existence of Hermitian positive definite solutions. In [6], Ivanov discussed sufficient conditions for existence the minimal $X_{s}$ and special $X_{l}$ positive definite solutions are derived and iterative procedures for computing these solutions. The problem in the Hermitian positive definite solutions of the matrix equation has been extensively researched in [7-12].

Throughout this paper, let $\|\cdot\|$ denote the spectral norm for square matrices unless otherwise noted, that is, $\|A\|=\sqrt{\max _{i} \lambda_{i}}$, where the $\lambda_{i}$ are the eigenvalues of $A A^{*}, I$ the identity matrix, and $A^{*}$ the conjugate transpose of $A$. The notation $X>0$ means that $X$ is a positive definite Hermitian matrix, $A>B$ is used to indicate that $A-B>0$, that is, $A-B$ be Hermitian positive definite.

In this paper, motivated by the results mentioned earlier, we give some necessary and sufficient conditions for the existence of a Hermitian positive definite solution of (1.1), and these necessary and sufficient conditions are different to $[3,5]$. Based on them, we also present some properties of the coefficient matrix $A$ are presented and two equivalent equations of (1.1) when the matrix equation has a Hermitian positive definite solution.

\section{Preliminaries and Lemmas}

The following are several basic properties and are useful in this paper.

Lemma 2.1. The spectral norm is monotonic norm, that is, if $0<A \leq B$, then $\|A\| \leq\|B\|$.

Lemma 2.2. If $M$ is positive definite Hermitian matrix, then there is a unique positive definite Hermitian matrix $W$ such that $M=W^{2}$.

Lemma 2.3 (see [3, Corollary 2.1]). If $\mathrm{X}$ is a positive definite solution of (1.1), then

$$
I<X<I+A^{*} A
$$


Lemma 2.4 (see [13, Theorem 4]). If (1.1) has a solution, then

$$
\|A\|<1
$$

Moreover, if $A$ has order $>2$, then $\|A\|$ can take any value in the interval $[0,1)$ for $(1.1)$ to have a solution.

\section{The Main Results}

In this section, we present our main results.

Theorem 3.1. The matrix equation (1.1) has a Hermitian positive definite solution $X$ if and only if A unitary equivalent to $\Lambda^{2} Z$, that is, $A$ can be factorized as

$$
A=U^{*} \Lambda^{2} Z U
$$

where $\Lambda=\operatorname{diag}\left(\sqrt{\lambda_{1}}, \sqrt{\lambda_{2}}, \ldots, \sqrt{\lambda_{s}}\right), \lambda_{i}$ are the eigenvalues of $X, i=1,2, \ldots, n, X=U^{*} \Lambda^{2} U$, and the columns of

$$
\left(\begin{array}{l}
\Lambda \\
Z
\end{array}\right)
$$

are orthonormal. In this case $X=U^{*} \Lambda^{2} U$ is a Hermitian positive definite solution of (1.1) and all Hermitian positive definite solutions of (1.1) can be formed in this way. By the result mentioned earlier, the solving of (1.1) can be transformed to the solving of the equation

$$
\Lambda_{1}+C^{*} \Lambda_{1}^{-2} C=I
$$

where $C=U A U^{*}$ is a nonsingular Hermitian matrix, $\Lambda_{1}=\operatorname{diag}\left(\lambda_{1}, \lambda_{2}, \ldots, \lambda_{s}\right), \lambda_{i}$ are the eigenvalues of $X, i=1,2, \ldots, n$.

Proof. If the matrix equation (1.1) has a Hermitian positive definite solution $X$, then there exists a unitary matrix $U$ satisfied $X=U^{*} \Lambda_{1} U$ where $\Lambda_{1}=\operatorname{diag}\left(\lambda_{1}, \lambda_{2}, \ldots, \lambda_{n}\right), \lambda_{i}(i=$ $1,2, \ldots, n)$ are the eigenvalues of $X$. Let $\Lambda_{1}=\Lambda^{2}$, then $\Lambda=\operatorname{diag}\left(\sqrt{\lambda_{1}}, \sqrt{\lambda_{2}}, \ldots, \sqrt{\lambda_{s}}\right)$, we rewrite the matrix equation (1.1) as

$$
U^{*} \Lambda^{2} U+A^{*}\left(U^{*} \Lambda^{2} U\right)^{-2} A=I
$$

so

$$
U^{*} \Lambda^{2} U+A^{*} U^{*} \Lambda^{-4} U A=I .
$$


Because $U$ is a unitary matrix, hence

$$
\Lambda^{2}+\left(U^{*}\right)^{-1} A^{*} U^{*} \Lambda^{-4} U A U^{-1}=\left(U^{*}\right)^{-1} I U^{-1}=I .
$$

Thus

$$
\left(\begin{array}{c}
\Lambda \\
\Lambda^{-2} U A U^{*}
\end{array}\right)^{*}\left(\begin{array}{c}
\Lambda \\
\Lambda^{-2} U A U^{*}
\end{array}\right)=I
$$

Let $Z=\Lambda^{-2} U A U^{*}$, then $A=U^{*} \Lambda^{2} Z U$ and by (3.3), it is easy to see that the columns of

$$
\left(\begin{array}{l}
\Lambda \\
Z
\end{array}\right)
$$

are orthonormal.

Conversely, suppose that $A$ has the decomposition (3.1) and $X=U^{*} \Lambda^{2} U$, where $U$ is a unitary matrix. Since the columns of

$$
\left(\begin{array}{l}
\Lambda \\
Z
\end{array}\right)
$$

are orthonormal, so

$$
\left(\begin{array}{c}
\Lambda \\
\Lambda^{-2} U A U^{*}
\end{array}\right)^{*}\left(\begin{array}{c}
\Lambda \\
\Lambda^{-2} U A U^{*}
\end{array}\right)=I
$$

that is,

$$
\Lambda^{2}+\left(U^{*}\right)^{-1} A^{*} U^{*} \Lambda^{-4} U A U^{-1}=\left(U^{*}\right)^{-1} I U^{-1}=I
$$

Then, we have

$$
\begin{aligned}
X+A^{*} X^{-2} A & =U^{*} \Lambda^{2} U+A^{*}\left(U^{*} \Lambda U\right)^{-4} A \\
& =U^{*} \Lambda U+\left(U^{*} \Lambda^{2} Z U\right)^{*} U^{*} \Lambda^{-4} U U^{*} \Lambda^{2} Z U \\
& =I .
\end{aligned}
$$

Hence $X$ is a Hermitian positive definite solution of (1.1).

The proof of Theorem 3.1 is complete. 
Theorem 3.2. If the matrix equation (1.1) has a Hermitian positive definite solution $\mathrm{X}$, then

$$
\|A\|^{1 / 2}<\bar{\lambda}<1, \quad \underline{\imath}<1-\|A\|,
$$

where $\bar{\lambda}=\max \left\{\lambda_{i}, i=1,2, \ldots, n\right\}, \underline{\lambda}=\min \left\{\lambda_{i}, i=1,2, \ldots, n\right\}, \lambda_{i}, i=1,2, \ldots, n$ are the eigenvalues of $X$.

Proof. By Theorem 3.1, if $\Lambda_{1}$ is a Hermitian positive definite solution of (3.3), then we have

$$
\Lambda_{1}>0, \quad C^{*} \Lambda_{1}^{-2} C>0,
$$

thus

$$
C^{*} \Lambda_{1}^{-2} C=I-\Lambda_{1}>0
$$

hence $I-\Lambda_{1}>0$, that is,

$$
0<\lambda_{i}<1
$$

where $\lambda_{i}, i=1,2, \ldots, n$.

Without loss of generality, suppose $0<\lambda_{1}<\lambda_{2}<\cdots<\lambda_{n}<1$, then $\bar{\lambda}=\lambda_{n}<1$. By the proof of Theorem 3.1, we have

$$
\left(\Lambda_{1}^{-1} U A\right)^{*}\left(\Lambda_{1}^{-1} U A\right)=U^{*}\left(I-\Lambda_{1}\right) U
$$

Let $\Sigma=\operatorname{diag}\left(\sigma_{1}, \sigma_{2}, \ldots, \sigma_{n}\right) \geq 0$ such that $\Lambda_{1}+\Sigma^{2}=I$, where $\sigma_{1} \geq \sigma_{2} \geq \cdots \geq \sigma_{n}$. Since $\bar{\lambda}<1$, then $\Sigma=\operatorname{diag}\left(\sigma_{1}, \sigma_{2}, \ldots, \sigma_{n}\right)>0,1>\sigma_{1} \geq \sigma_{2} \geq \cdots \geq \sigma_{n}>0$, and $\sigma_{1}=\sqrt{1-\lambda_{1}}$, that is,

$$
\sigma_{1}=\sqrt{1-\underline{\lambda}}
$$

Now let

$$
B=\Lambda_{1}^{-1} U A U^{*}=\left(b_{1}, b_{2}, \ldots, b_{n}\right),
$$

where $b_{i}$ is $n$-column vector, then $B^{*} B=\sum$, so $B$ is column orthogonal and $b_{i}^{*} b_{i}=\sigma_{i}^{2}, i=$ $1,2, \ldots, n$. Let $v_{i}=b_{i} / \sigma_{i}, i=1,2, \ldots, n$, and with CS factorization theorem, extend them to form an orthogonal basis of $C^{n}$. Thus $V=\left(v_{1}, v_{2}, v_{n}\right)$ is a unitary matrix such that $B=V \sum$, and hence

$$
A=U^{*} \Lambda_{1} V \sum U
$$


So

$$
\begin{aligned}
\|A\| & =\left\|U^{*} \Lambda_{1} V \sum U\right\| \\
& =\left\|\Lambda_{1} V \sum\right\| \\
& \leq\left\|\Lambda_{1}\right\|\left\|\sum\right\| \\
& =\bar{\jmath}^{2}(1-\underline{\jmath}),
\end{aligned}
$$

thus we have

$$
\|A\| \leq \bar{\lambda}^{2}
$$

that is,

$$
\begin{gathered}
\|A\|^{1 / 2} \leq \bar{\lambda} \\
\|A\| \leq 1-\underline{\lambda}
\end{gathered}
$$

that is,

$$
\underline{\imath} \leq 1-\|A\| .
$$

The proof of Theorem 3.2 is complete.

Theorem 3.3. If the matrix equation (1.1) has a Hermitian positive definite solution $X$, let $\alpha=$ $\max \left\{\lambda_{i}^{2}, i=1,2, \ldots, n\right\}, \beta=\max \left\{1-\lambda_{i}, i=1,2, \ldots, n\right\}, \underline{\lambda}=\min \left\{\lambda_{i}, i=1,2, \ldots, n\right\}$, where $\lambda_{i}, i=1,2, \ldots, n$ are the eigenvalues of $X$, then

(1) $A^{*} A<\beta \Lambda^{2}<\beta \alpha I$;

(2) $\underline{\lambda} I<\Lambda<I-\alpha A^{*} A$.

Proof. By Theorem 3.1, $C=U A U^{*}$, where $U$ is a unitary matrix, hence $A^{*} A=U C^{*} C U^{*}$.

Let $\alpha=\max \left\{\lambda_{i}^{2}, i=1,2, \ldots, n\right\}, \beta=\max \left\{1-\lambda_{i}, i=1,2, \ldots, n\right\}, \underline{\lambda}=\min \left\{\lambda_{i}, i=\right.$ $1,2, \ldots, n\}$, where $\lambda_{i}, i=1,2, \ldots, n$ be the eigenvalues of $X$.

(1) By Theorem 3.1, $\Lambda_{1}$ is a Hermitian positive definite solution of (3.3), thus

$$
\Lambda<\alpha I, \quad C^{*} \Lambda_{1}^{-2} C=I-\Lambda_{1}<I,
$$

hence

$$
C^{*} \Lambda_{1}^{-2} C=\left(I-\Lambda_{1}\right)<\beta I, \quad \Lambda_{1}^{2}<\alpha I<I
$$


So

$$
A^{*} A=U^{*} C^{*} C U<\beta U^{*} \Lambda_{1}^{2} U<\alpha \beta I .
$$

(2) Since $\Lambda_{1}$ is a Hermitian positive definite solution of (3.3), $C$ is a nonsingular matrix, that is, $C^{*} \Lambda_{1}^{-2} C=I-\Lambda_{1}$, by (1) $\Lambda_{1}^{-2}>\alpha I$, then

$$
C^{*} \Lambda_{1}^{-2} C>\alpha C^{*} C
$$

so

$$
\alpha A^{*} A=U \alpha C^{*} C U^{*}<I-\Lambda_{1}
$$

thus we have

$$
\underline{\lambda} I<\Lambda_{1}<I-\alpha A^{*} A .
$$

The proof of Theorem 3.3 is complete.

Theorem 3.4. Suppose $A$ and $B$ are two positive definite Hermitian matrices, and $A>B$, then there exist a nonsingular matrices $T$ which satisfied

(1)

$$
T^{*} A T=I, \quad T^{*} B T=\Lambda,
$$

where $\Lambda=\operatorname{diag}\left(\lambda_{1}, \lambda_{2}, \ldots, \lambda_{n}\right), \lambda_{i}$ are the eigenvalues of $A^{-1} B, i=1,2, \ldots, n$;

(2) $0<\lambda_{i}<1$, and the columns of

$$
\left(\begin{array}{c}
\Lambda^{1 / 2} \\
(A-B)^{1 / 2} T
\end{array}\right)
$$

are orthonormal, $i=1,2, \ldots, n$.

Proof. (1) Since the matrix $A$ is positive definite Hermitian, then there exists a unique positive definite Hermitian matrix $W$ such that $A=W^{2}$. We note

$$
F=\left(W^{-1}\right)^{*} B W^{-1}=W B W^{-1} .
$$

It is to prove that $F$ is Hermitian matrix also, so there exists a unitary matrix $U$ such that $U^{*} F U=\Lambda$, where $\Lambda=\operatorname{diag}\left(\lambda_{1}, \lambda_{2}, \ldots, \lambda_{n}\right), \lambda_{i}$ are the eigenvalues of $F$. Hence we have the following equation

$$
U^{*}\left(W^{-1}\right)^{*} B W^{-1} U=\Lambda
$$


Let $T=W^{-1} U$, then $T$ is nonsingular. Since

$$
\begin{aligned}
\operatorname{det}(\lambda I-F) & =\frac{\operatorname{det}(W(\lambda I-F) W)}{\operatorname{det}(A)} \\
& =\frac{\operatorname{det}\left(\lambda W^{*} W-W^{*} F W\right)}{\operatorname{det}(A)} \\
& =\frac{\operatorname{det}(\lambda A-B)}{\operatorname{det}(A)} \\
& =\frac{\operatorname{det}\left(\lambda A-A A^{-1} B\right)}{\operatorname{det}(A)} \\
& =\operatorname{det}\left(\lambda I-A^{-1} B\right),
\end{aligned}
$$

so the eigenvalue of $A^{-1} B$ is equal to the eigenvalue of $F$.

(2) If (3.31) is hold, then we have

$$
T^{*}(A-B) T=I-\Lambda
$$

Since $A>B, T$ is nonsingular, so $T^{*}(A-B) T$ is positive definite Hermitian.

Thus $I-\Lambda>0$, that is, $0<\lambda_{i}<1, i=1,2, \ldots, n$. It is easy to see that the columns of

$$
\left(\begin{array}{c}
\Lambda^{1 / 2} \\
(A-B)^{1 / 2} T
\end{array}\right)
$$

are orthonormal.

The proof of Theorem 3.4 is complete.

Theorem 3.5. The matrix equation (1.1) has a Hermitian positive definite solution $X$ if and only if A has the following factorization:

$$
A=T \Lambda^{-1 / 2} Z
$$

where $\Lambda=\operatorname{diag}\left(\lambda_{1}, \lambda_{2}, \ldots, \lambda_{n}\right), \lambda_{i}, i=1,2, \ldots, n$ are the eigenvalues of $X^{-3}, T^{*} X T=I, T^{*} X^{-2} T=$ $\Lambda$ and the columns of

$$
\left(\begin{array}{c}
T^{-1} \\
Z
\end{array}\right)
$$

are orthonormal. In this case $\mathrm{X}=\left(T^{-1}\right)^{*} T^{-1}$ is a Hermitian positive definite solution and all Hermitian positive definite solutions can be formed in this way. 
Proof. If the matrix equation (1.1) has a Hermitian positive definite solution $X$, then by Theorem 3.3, there exists a nonsingular matrix $T$ which satisfied

$$
T^{*} X T=I, \quad T^{*} X^{-2} T=\Lambda,
$$

hence

$$
X=\left(T^{-1}\right)^{*} T^{-1}, \quad X^{-2}=\left(T^{-1}\right)^{*} \Lambda T^{-1}
$$

So (1.1) is equivalent to

$$
\left(T^{-1}\right)^{*} T^{-1}+A^{*}\left(T^{-1}\right)^{*} \Lambda T^{-1} A=I,
$$

or equivalently

$$
\left(\begin{array}{c}
T^{-1} \\
\Lambda^{1 / 2} T^{-1} A
\end{array}\right)^{*}\left(\begin{array}{c}
T^{-1} \\
\Lambda^{1 / 2} T^{-1} A
\end{array}\right)=I
$$

Let $\Lambda^{1 / 2} T^{-1} A=Z$, then $A=T \Lambda^{-1 / 2} Z$ and (3.43) means that the columns of

$$
\left(\begin{array}{c}
T^{-1} \\
\Lambda^{1 / 2} T^{-1} A
\end{array}\right)
$$

are orthonormal.

Conversely, suppose that $A$ has the decomposition $A=T \Lambda^{-1 / 2} Z$ and $X=\left(T^{-1}\right)^{*} T^{-1}$, where $U$ is a unitary matrix. Since the columns of

$$
\left(\begin{array}{c}
T^{-1} \\
Z
\end{array}\right)
$$

are orthonormal, so

$$
\left(\begin{array}{c}
T^{-1} \\
\Lambda^{1 / 2} T^{-1} A
\end{array}\right)^{*}\left(\begin{array}{c}
T^{-1} \\
\Lambda^{1 / 2} T^{-1} A
\end{array}\right)=I .
$$

Hence

$$
\left(T^{-1}\right)^{*} T^{-1}+A^{*}\left(T^{-1}\right)^{*} \Lambda T^{-1} A=I .
$$

Then we have

$$
X+A^{*} X^{-2} A=\left(T^{-1}\right)^{*} T^{-1}+\left(T^{-1}\right)^{*} \Lambda T^{-1}=I .
$$

The proof of Theorem 3.5 is complete. 
Theorem 3.6. The matrix equation (1.1) has a Hermitian positive definite solution $X$ if and only if $A$ has the following factorization:

$$
A=T Z,
$$

where $\Lambda=\operatorname{diag}\left(\lambda_{1}, \lambda_{2}, \ldots, \lambda_{n}\right), \lambda_{i}, i=1,2, \ldots, n$ are the eigenvalues of $X^{3}, T^{*} X T=\Lambda, T^{*} X^{-2} T=$ $I$ and the columns of

$$
\left(\begin{array}{c}
\Lambda^{1 / 2} T^{-1} \\
Z
\end{array}\right)
$$

are orthonormal. In this case $X=\left(T^{-1}\right)^{*} \Lambda T^{-1}$ is a Hermitian positive definite solution and all Hermitian positive definite solutions can be formed in this way.

Proof. If the matrix equation (1.1) has a Hermitian positive definite solution $X$, then by Theorem 3.3, there exists a nonsingular matrix $T$ which satisfied

$$
T^{*} X T=\Lambda, \quad T^{*} X^{-2} T=I,
$$

hence

$$
X=\left(T^{-1}\right)^{*} \Lambda T^{-1}, \quad X^{-2}=\left(T^{-1}\right)^{*} T^{-1}
$$

So (1.1) is equivalent to

$$
\left(T^{-1}\right)^{*} \Lambda T^{-1}+A^{*}\left(T^{-1}\right)^{*} T^{-1} A=I,
$$

or equivalently

$$
\left(\begin{array}{c}
\Lambda^{1 / 2} T^{-1} \\
T^{-1} A
\end{array}\right)^{*}\left(\begin{array}{c}
\Lambda^{1 / 2} T^{-1} \\
T^{-1} A
\end{array}\right)=I .
$$

Let $T^{-1} A=Z$, then $A=T Z$, and (3.54) means that the columns of

$$
\left(\begin{array}{c}
\Lambda^{1 / 2} T^{-1} \\
T^{-1} A
\end{array}\right)
$$

are orthonormal.

Conversely, suppose that $A$ has the decomposition $A=T Z$ and $X=\left(T^{-1}\right)^{*} \Lambda T^{-1}$, where $U$ is a unitary matrix. Since the columns of

$$
\left(\begin{array}{c}
\Lambda^{1 / 2} T^{-1} \\
Z
\end{array}\right)
$$


are orthonormal, then

$$
\left(\begin{array}{c}
\Lambda^{1 / 2} T^{-1} \\
T^{-1} A
\end{array}\right)^{*}\left(\begin{array}{c}
\Lambda^{1 / 2} T^{-1} \\
T^{-1} A
\end{array}\right)=I .
$$

Thus

$$
\left(T^{-1}\right)^{*} \Lambda T^{-1}+A^{*}\left(T^{-1}\right)^{*} T^{-1} A=I .
$$

Then we have

$$
X+A^{*} X^{-2} A=\left(T^{-1}\right)^{*} \Lambda T^{-1}+\left(T^{-1}\right)^{*} T^{-1}=I .
$$

The proof of Theorem 3.6 is complete.

Theorem 3.7. The matrix equation (1.1) has a Hermitian positive definite solution $X$ if and only if $\Lambda T$ is unitary, where $\Lambda=\sqrt{\Lambda_{1}+I}, \Lambda_{1}=\operatorname{diag}\left(\lambda_{1}, \lambda_{2}, \ldots, \lambda_{n}\right), \lambda_{i}, i=1,2, \ldots, n$ are the eigenvalues of $X^{-1} A^{*} X^{-2} A$.

Proof. If the matrix equation (1.1) has a Hermitian positive definite solution $X$, then by Theorem 3.3, there exists a nonsingular matrix $T$ which satisfied

$$
T^{*} X T=I, \quad T^{*} A^{*} X^{-2} A T=\Lambda_{1},
$$

hence

$$
X=\left(T^{-1}\right)^{*} T^{-1}, \quad A^{*} X^{-2} A=\left(T^{-1}\right)^{*} \Lambda_{1} T^{-1} .
$$

So (1.1) is equivalent to

$$
\left(T^{-1}\right)^{*} T^{-1}+\left(T^{-1}\right)^{*} \Lambda_{1} T^{-1}=I,
$$

that is,

$$
\left(T^{-1}\right)^{*}\left(I+\Lambda_{1}\right) T^{-1}=I .
$$

Let $\Lambda=\sqrt{\Lambda_{1}+I}$, then (3.63) is equivalent to

$$
\left(T^{-1}\right)^{*} \Lambda^{2} T^{-1}=I
$$


So

$$
\left(\Lambda T^{-1}\right)^{*}\left(\Lambda T^{-1}\right)=I
$$

Thus $\Lambda T$ is unitary.

The proof of Theorem 3.7 is complete.

Similarly, we have the following theorem.

Theorem 3.8. The matrix equation (1.1) has a Hermitian positive definite solution $X$ if and only if $\Lambda T$ is unitary, where $\Lambda=\sqrt{\Lambda_{1}+I}, \Lambda_{1}=\operatorname{diag}\left(\lambda_{1}, \lambda_{2}, \ldots, \lambda_{n}\right), \lambda_{i}, i=1,2, \ldots, n$ are the eigenvalues of $A^{-1} X^{2}\left(A^{-1}\right)^{*} X$.

Remark 3.9. By Theorems 3.7 and 3.8, the solving of (1.1) is transformed to the solving of

$$
T^{*} T=\Lambda,
$$

where $\Lambda=\operatorname{diag}\left(\lambda_{1}-1, \lambda_{2}-1, \ldots, \lambda_{n}\right)-1, \lambda_{i}, i=1,2, \ldots, n$ are the eigenvalues of $A^{-1} X^{2}\left(A^{-1}\right)^{*} X$ or $X^{-1} A^{*} X^{-2} A$ is a nonsingular Hermitian matrix.

It is easy to see that every eigenvalue $\lambda_{i}$ of $A^{-1} X^{2}\left(A^{-1}\right)^{*} X$ or $X^{-1} A^{*} X^{-2} A$ satisfied

$$
\lambda_{i}>1
$$

\section{Acknowledgment}

This research was supported by KJM of Shandong University of Technology Grant (2005KJM30).

\section{References}

[1] S. Huanfeng, L. Pingxiang, and Z. Liangpei, "A regularized super-resolution image reconstruction method," Journal of Image and Graphics, vol. 10, pp. 436-440, 2005.

[2] B. L. Buzbee, G. H. Golub, and C. W. Nielson, "On direct methods for solving Poisson's equations," SIAM Journal on Numerical Analysis, vol. 7, pp. 627-656, 1970.

[3] I. G. Ivanov, V. I. Hasanov, and B. V. Minchev, “On matrix equations $X \pm A^{*} X^{-2} A=I$," Linear Algebra and Its Applications, vol. 326, no. 1-3, pp. 27-44, 2001.

[4] A. Ferrante and B. C. Levy, "Hermitian solutions of the equation $X=Q+N X^{-1} N^{*}$," Linear Algebra and Its Applications, vol. 247, pp. 359-373, 1996.

[5] M. Cheng, "Necessary and sufficient conditions for the existence of a positive definite solution of the matrix equation $X+A^{*} X^{-2} A=I$," Acta Scientiarum Naturalium Universitatis Pekinensis, vol. 41, no. 1, pp. 55-61, 2005.

[6] I. G. Ivanov, "On positive definite solutions of the family of matrix equations $X+A^{*} X^{-n} A=Q$," Journal of Computational and Applied Mathematics, vol. 193, no. 1, pp. 277-301, 2006.

[7] R. A. Horn and C. R. Johnson, Topics in Matrix Analysis, Cambridge University Press, Cambridge, UK, 1991.

[8] S. Fital and C.-H. Guo, "A note on the fixed-point iteration for the matrix equations $X A^{*} X^{-1} A=I$," Linear Algebra and Its Applications, vol. 429, no. 8-9, pp. 2098-2112, 2008.

[9] S. M. El-Sayed and A. M. Al-Dbiban, "A new inversion free iteration for solving the equation $X A^{*} X^{-1} A=Q, "$ Journal of Computational and Applied Mathematics, vol. 181, no. 1, pp. 148-156, 2005. 
[10] S. M. El-Sayed and M. El-Alem, "Some properties for the existence of a positive definite solution of matrix equation $X+A X^{-2^{m}} A=I, "$ Applied Mathematics and Computation, vol. 128, no. 1, pp. 99-108, 2002.

[11] X. Zhan, "Computing the extremal positive definite solutions of a matrix equation," SIAM Journal on Scientific Computing, vol. 17, no. 5, pp. 1167-1174, 1996.

[12] S. M. El-Sayed and A. C. M. Ran, "On an iteration method for solving a class of nonlinear matrix equations," SIAM Journal on Matrix Analysis and Applications, vol. 23, no. 3, pp. 632-645, 2001.

[13] I. G. Ivanov and S. M. El-Sayed, "Properties of positive definite solutions of the equation $X+A^{*} X^{-2} A=$ I," Linear Algebra and Its Applications, vol. 279, no. 1-3, pp. 303-316, 1998. 


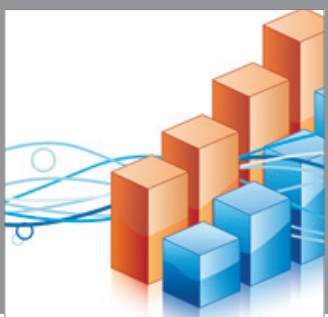

Advances in

Operations Research

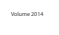

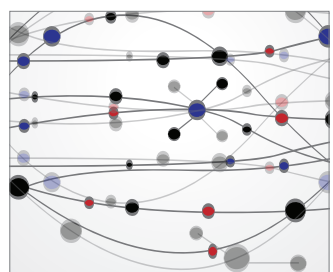

\section{The Scientific} World Journal
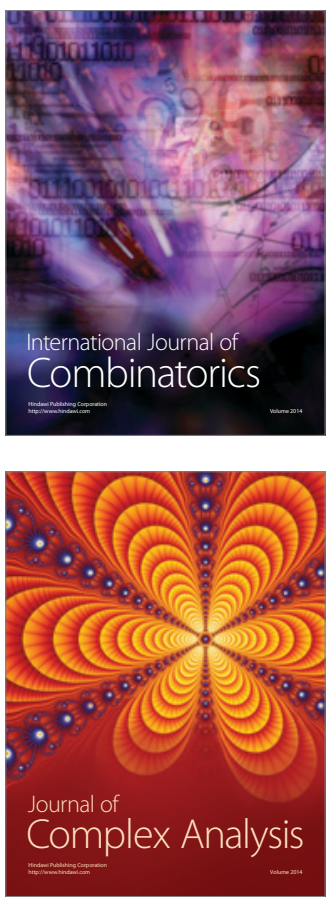

International Journal of

Mathematics and

Mathematical

Sciences
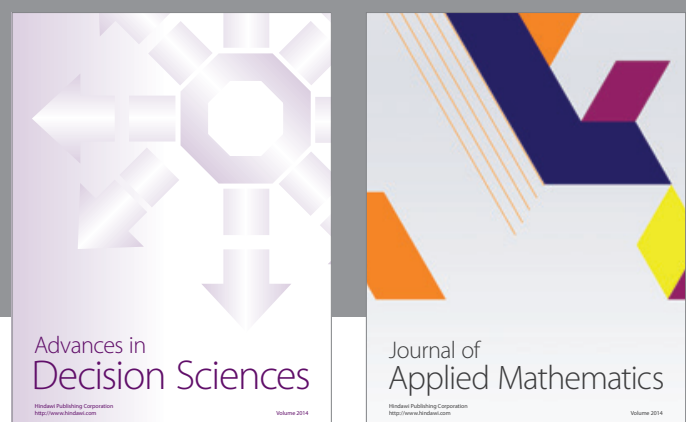

Journal of

Applied Mathematics
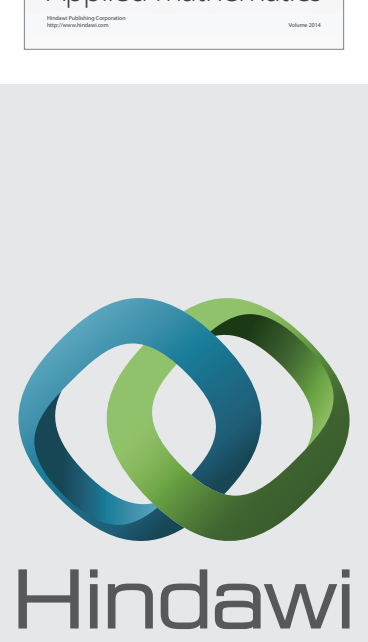

Submit your manuscripts at http://www.hindawi.com
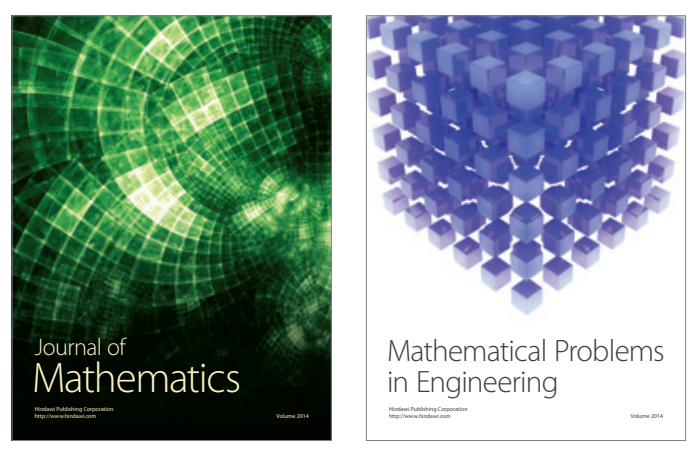

Mathematical Problems in Engineering
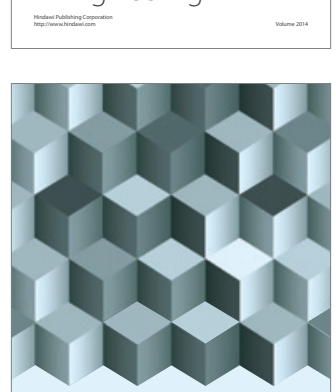

Journal of

Function Spaces
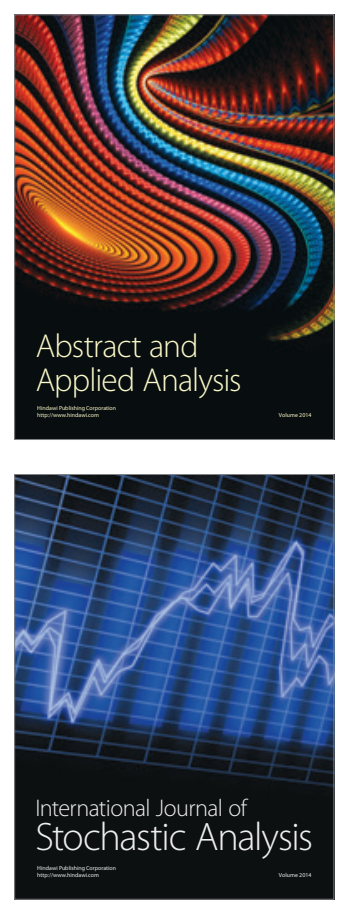

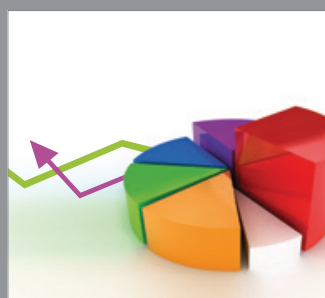

ournal of

Probability and Statistics

Promensencen
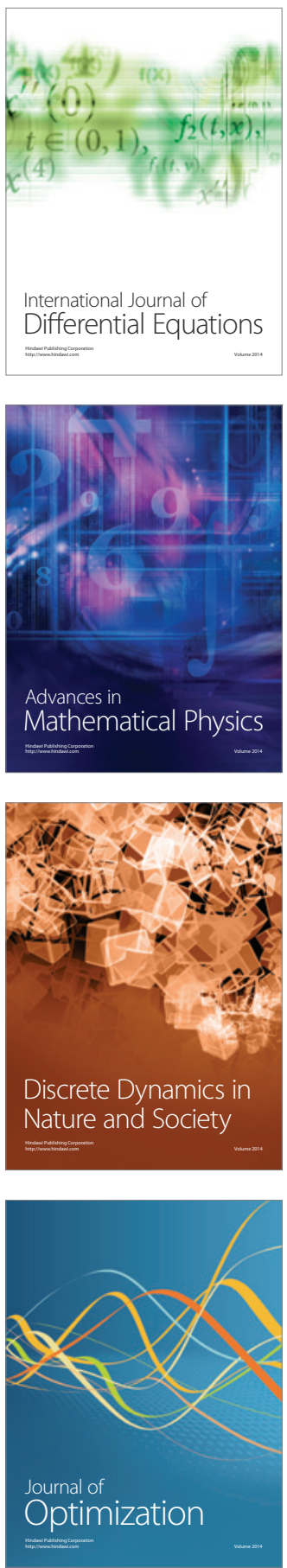